\title{
Multicenter evaluation of the hemolysis index in automated clinical chemistry systems
}

\author{
Giuseppe Lippi ${ }^{1,9,10, *}$, Gian Luca Salvagno ${ }^{1}$, \\ Norbert Blanckaert ${ }^{2,9}$, Davide Giavarina ${ }^{3}$, Sol \\ Green $^{4,9}$, Steve Kitchen ${ }^{5,9}$, Vladimir Palicka ${ }^{6,9}$, \\ Anne J. Vassault ${ }^{7,9}$ and Mario Plebani ${ }^{8-10}$ \\ ${ }^{1}$ Clinical Chemistry Laboratory, University of \\ Verona, Verona, Italy \\ ${ }^{2}$ Laboratory Medicine, University Hospital Leuven, \\ Leuven, Belgium \\ ${ }^{3}$ Clinical Chemistry Laboratory, San Bortolo \\ Hospital, Vicenza, Italy \\ ${ }^{4}$ BD Diagnostics - Preanalytical Systems, New \\ Jersey, USA \\ ${ }^{5}$ Sheffield Hemophilia and Thrombosis Center, \\ Royal Hallamshire Hospital, Sheffield, England \\ ${ }^{6}$ Institute of Clinical Biochemistry and Diagnostics, \\ Charles University, University Hospital, Hradec \\ Kralove, Czech Republic \\ ${ }^{7}$ Laboratoire de Biochimie B Hôpital Necker Enfants \\ Malades, AP-HP, Paris, France \\ ${ }^{8}$ Department of Laboratory Medicine, University \\ of Padova, Padova, Italy \\ ${ }^{9}$ EPSC - European Preanalytical Scientific \\ Committee (www.specimencare.com) \\ ${ }^{10}$ International Federation of Clinical Chemistry and \\ Laboratory Medicine Working Group on Patient's \\ Safety
}

\begin{abstract}
Background: In vitro hemolysis, the prevailing cause of preanalytical error in routine laboratory diagnostics, might influence the reliability of several tests, affect the quality of the total testing process and jeopardize patient safety. Although laboratory instrumentation is now routinely equipped with systems capable of automatically testing and eventually correcting for hemolysis interference, to our knowledge there are no reports that have compared the efficiency of different analytical platforms for identifying and classifying specimens with hemolysis.

Methods: Serum from a healthy volunteer was spiked with varying amounts of hemolyzed blood from the same volunteer, providing a serum free hemoglobin concentration ranging from $0.0 \mathrm{~g} / \mathrm{L}$ to $2.0 \mathrm{~g} / \mathrm{L}$ as measured by the reference cyanmethemoglobin assay. The spiked serum samples were shipped to seven sepa-

*Corresponding author: Prof. Giuseppe Lippi, MD, Sezione di Chimica e Microscopia Clinica, Dipartimento di Scienze Morfologico-Biomediche, Università degli Studi di Verona, Ospedale Policlinico G.B. Rossi, Piazzale Scuro, 10, 37134 Verona, Italy

Phone: +39-045-8124308, Fax: +39-045-8201889,

E-mail: ulippi@tin.it; giuseppe.lippi@univr.it

Received March 24, 2009; accepted May 6, 2009;

previously published online June 24, 2009
\end{abstract}

rate laboratories and the hemolysis index $(\mathrm{HI})$ was tested in triplicate on the following analytical platforms: Roche Modular System $P(n=4)$ and Integra 400 Plus $(n=1)$, Siemens Dimension $\operatorname{RxL}(n=3)$, ADVIA $2400(n=1)$ and ADVIA $1800(n=1)$, Olympus AU $680(n=1)$ and Coulter DXC $800(n=1)$.

Results: Satisfactory agreement of $\mathrm{HI}$ results was observed among the various analytical platforms, despite a trend toward overestimation by the ADVIA 2400 and 1800. After normalizing results according to the instrument-specific alert value, discrepancies were considerably reduced. All instruments except for the Dimension $R x L$ gave values normalized to the instrument-specific alert value, $<1.0$ for the sample with $0.048 \mathrm{~g} / \mathrm{L}$ free hemoglobin, and $>1.0$ for the sample with $0.075 \mathrm{~g} / \mathrm{L}$ free hemoglobin. The results of the four Modular System P tests were also highly reproducible among the different facilities. When evaluating instruments that provided quantitative $\mathrm{HI}$ results, the mean intra-assay coefficient of variation (CV) calculated for the triplicate determinations was always between $0.1 \%$ and $2.7 \%$.

Conclusions: The results of this multicenter evaluation confirm that efficiency of different analytical platforms to correctly identify and classify unsuitable samples is satisfactory. However, more effort should be placed on the standardization of reporting HI. All the instruments that we tested provide either quantitative or qualitative results that are essentially comparable, but which should always be compared with the instrument-specific alert values to harmonize their efficiency.

Clin Chem Lab Med 2009;47:934-9.

Keywords: errors; hemolysis; hemolysis index (HI); patient safety; preanalytical variability.

\section{Introduction}

Preanalytical variability is a major problem in laboratory diagnostics because several phases of this process can affect specimen integrity and the reliability of test results. Spurious test results obtained on unsuitable specimens not only impact the quality of the total testing process, but might produce adverse clinical and economic outcomes. Several lines of evidence show that in vitro hemolysis is the most prevalent preanalytical error; its frequency reportedly affecting as many as $3.3 \%$ of routine samples referred to the clinical laboratory, and accounting for up to $70 \%$ of all the unsuitable specimens received (1-7). Hemolysis is commonly defined as the release of hemoglobin from erythrocytes into the surrounding plasma as a result of damage or breakdown of the 
cell membrane. Hemolysis confers a pink to red hue coloration of the plasma or serum following centrifugation of specimens. Traditionally, the upper reference limit for free hemoglobin varies from 0.02 $\mathrm{mg} / \mathrm{L}$ (plasma) to $0.05 \mathrm{mg} / \mathrm{L}$ (serum), and it becomes visually detectable when such concentration exceeds $0.3-0.6 \mathrm{~g} / \mathrm{L}(18.8-37.6 \mu \mathrm{mol} / \mathrm{L})$, corresponding roughly to $0.5 \%-1.0 \%$ of lysed erythrocytes (7).

The interference effect from hemolysis in laboratory diagnostics is a consequence of several coexisting biological and analytical causes, including (a) leakage of hemoglobin and other intracellular components into the surrounding fluid thereby producing false elevations of intracellular analytes or dilutional effects, (b) method- and analyte-dependent spectrophotometric interferences and (c) chemical interference in a variety of analytic reactions. At high concentrations of serum hemoglobin, all these interference effects might coexist, thereby producing spurious variations that do not necessarily follow the same pattern resulting in overestimation or underestimation $(1,2,7,8)$. The magnitude of this problem is further magnified by the management of hemolytic specimens in the laboratory, from their identification to the optimal means of handling them. One of the more debated issues is the identification of hemolysis by laboratory personnel, because hemolysis in serum or plasma is undetectable until the specimen has been centrifuged. However, even following centrifugation there are no definitive guidelines, other than recommendations or suggestions, that define what means should be used to assess the degree of hemolysis or what thresholds should be used to guide the rejection of samples (7).

In vitro hemolysis has traditionally been assessed on an arbitrary basis through visual inspection by laboratory personnel. More recently, however, several preanalytical modules and analytical platforms have been equipped with systems capable of automatically testing and eventually correcting for a broad series of analytical interferences, including hemolysis. In most cases, the instruments report a qualitative or quantitative "hemolysis index" (HI), which should be compared with manufacturer-, instrument- and analyte-specific alert values before deciding whether to perform testing. This process is not intended for diagnostic purposes, but instead used to determine the condition of a sample. The user often can adjust the level at which the interference generates a flag, and also can customize the operating mode to reflect their own individual operating requirements for reporting interference. The implementation of this technology offers several advantages. It can overcome the inherent limits of visual inspection and it helps to improve the recognition of specimens with mild hemolysis ( $\sim 0.6 \mathrm{~g} / \mathrm{L}$ of free hemoglobin) which are difficult to detect by visual inspection but might still be unsuitable for the measurements of several analytes such as aspartate aminotransferase, lactate dehydrogenase and potassium (8). The index may also be useful as a quality assurance indicator to evaluate and improve the best preanalytical practices among different blood collection sites or hospital wards. Finally, the automated $\mathrm{HI}$ allows standardization and harmonization of behavior among operators in the same laboratory or among different facilities. Although widespread implementation of this technology is thus advantageous, advisable and even recommended ( 7 , $9)$, there are no reports, to the best of our knowledge, that have compared the efficiency of different analytical platforms for the identification and correct classification of hemolyzed specimens.

\section{Materials and methods}

The preparation of the samples was performed at a central location in the Clinical Chemistry Laboratory of the Verona University Hospital. On the morning of day $1,50 \mathrm{~mL}$ of blood were collected from a healthy volunteer into 10 siliconized vacuum tubes containing no additives (BD Vacutainer ${ }^{\circledR}$ Serum Glass Tubes, 5.0 mL; Cat\# 367614, Becton Dickinson UK Ltd, Plymouth, UK), using a BD Vacutainer ${ }^{\circledR}$ Multi-sample $21 \mathrm{G}$ needle (Becton Dickinson). One $5 \mathrm{~mL}$ specimen (hemolyzed sample $-5 \mathrm{~mL}$ of whole blood) was immediately stored at $-70^{\circ} \mathrm{C}$, whereas the other nine samples were centrifuged at $1500 \times g$ for $10 \mathrm{~min}$ at room temperature. The serum was separated and pooled from the nine tubes, creating $\sim 22 \mathrm{~mL}$ (pooled sample), and also stored at $-70^{\circ} \mathrm{C}$. On the morning of day 2 , the hemolysed sample and the pooled sample were thawed and re-centrifuged at $1500 \times g$ for $10 \mathrm{~min}$. Free hemoglobin was quantitated in the supernatant of the hemolyzed sample by the reference cyanmethemoglobin method using a UV-1700 spectrophotometer (Shimadzu Italia S.I.r., Milan, Italy) (10). Five serial dilutions of free serum hemoglobin were further prepared by mixing serial aliquots of hemolyzed sample and pooled sample to achieve final free hemoglobin concentrations ranging from $0.09 \mathrm{~g} / \mathrm{L}$ to $2.0 \mathrm{~g} / \mathrm{L}$ (labeled as Sample A, B, C, D and E). These concentrations approximately cover the degree of hemolysis seen in most hemolyzed samples encountered by clinical

Table 1 Description of centers and instrumentation.

\begin{tabular}{ll}
\hline Laboratory & Instruments $^{\text {a }}$ \\
\hline University Hospital, Verona, Italy & Roche Modular System P and Siemens Dimension RxL \\
University Hospital, Padova, Italy & Roche Modular System P and Siemens Dimension RxL \\
San Bortolo Hospital, Vicenza, Italy & Siemens ADVIA 2400 and 1800 \\
Hôpital Saint Antoine, Paris, France & Olympus AU 680 and Beckman Coulter DxC 800 \\
University Hospital, Leuven, Belgium & Roche Modular System P and Siemens Dimension RxL \\
University Hospital, Hradec Kralove, Czech Republic & Roche Modular System P \\
BD PAS, European Clinical Laboratory, Plymouth, UK & Roche Integra 400 Plus \\
\hline
\end{tabular}

${ }^{a}$ F. Hoffmann-La Roche Ltd., Basel, Switzerland; Siemens Healthcare Diagnostics Inc., Deerfield, IL, USA; Olympus Medical System Corp., Tokyo, Japan; Beckman Coulter Inc., Fullerton, CA, USA. 
Table 2 Description of the instrumentation used to test the hemolysis index ( $\mathrm{HI})$ and the instrument-specific alert values.

\begin{tabular}{lll}
\hline Manufacturer & Instrument & Alert value (free hemoglobin) \\
\hline Beckman & Coulter DxC 800 & 2 (arbitrary unit, corresponding to $0.50-1.0 \mathrm{~g} / \mathrm{L}$ ) \\
Olympus & AU 680 & " $+^{\prime \prime}$ (arbitrary unit, corresponding to $0.50-1.0 \mathrm{~g} / \mathrm{L}$ ) \\
Roche & Modular System P & $0.60 \mathrm{~g} / \mathrm{L}$ \\
Roche & Integra 400 PLUS & $0.60 \mathrm{~g} / \mathrm{L}$ \\
Siemens & RxL Dimension & 2 (arbitrary unit, corresponding to 0.25-0.50 g/L) \\
Siemens & ADVIA 2400 & $0.80 \mathrm{~g} / \mathrm{L}^{\mathrm{a}}$ \\
Siemens & ADVIA 1800 & $0.80 \mathrm{~g} / \mathrm{L}^{\mathrm{a}}$ \\
\hline
\end{tabular}

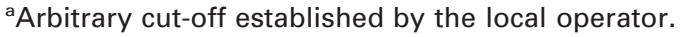

laboratories (4). For each of these separate samples, seven aliquots of $0.6 \mathrm{~mL}$ each were transferred into $1 \mathrm{~mL}$ plastic cups and shipped to the laboratories participating in this study (Table 1). Samples were kept stored until all laboratories had received their samples. The tests were performed simultaneously in the seven laboratories participating in the study. Before assessment, the samples were mixed manually by 5-6 complete inversions. The $\mathrm{HI}$ was tested on each sample (from A to $E$ ) in triplicate on the analytical platforms reported in Table 3 , and quantitative results were reported as mean $\pm \mathrm{SD}$. Concomitantly, the hemoglobin concentration of each sample was retested by the cyanmethemoglobin method in the Clinical Chemistry Laboratory of the Verona University Hospital. When quantitative results were available, results were normalized to the instrumentspecific alert value (i.e., measured value/alert value) as provided by the manufacturers (Table 2). In addition, an arbitrary threshold of free hemoglobin measured by the cyanmethemoglobin method was established at $0.6 \mathrm{~g} / \mathrm{L}$. This concentration of free hemoglobin is suggestive of a mildly hemolyzed specimen and represents the threshold for deciding whether some test results might already be significantly affected by in vitro hemolysis (e.g., aspartate aminotransferase, lactate dehydrogenase and potassium) (8).

\section{Results}

The results of this multicenter evaluation of the $\mathrm{HI}$ are shown in Tables 3 and 4 . No variation in the free hemoglobin concentration in the specimens was observed prior to and following shipment, as verified by the cyanmethemoglobin measurement (Passing and Bablok regression analysis and Pearson correlation coefficient: [before shipment] $=1.02 \times$ [after shipment] +0.01; $\quad r=1.00 ; \quad p<0.001)$. Satisfactory agreement was observed among the various analytical platforms, despite the presence of a trend toward overestimation by both the ADVIA 2400 and 1800 Dimension (Table 3). However, after normalizing results according to the instrument-specific alert value (i.e., [measured value]/[0.6 g/L] for the cyanmethemoglobin method, and [measured value]/ [highest alert value] for the instruments), such discrepancies were considerably reduced (Table 4). In particular, all instruments except for the RxL Dimension gave "normalized" values $<1.0$ for sample B (free hemoglobin value of $0.048 \mathrm{~g} / \mathrm{L}$ by the cyanmethemoglobin method) and $>1.0$ for sample $C$ (free hemoglobin value of $0.075 \mathrm{~g} / \mathrm{L}$ by the cyanmethemoglobin method). The quantitative $\mathrm{HI}$ results obtained on the Modular System $\mathrm{P}$ were also highly reproducible among the different facilities, as demonstrated by the non-significant variation $(p=0.911$ by KruskalWallis test) and the excellent correlation shown by Passing and Bablok regression analysis and the Pearson correlation coefficient (Table 5). The triplicate measurements using instruments providing semiquantitative $\mathrm{HI}$ results were always identical for all the samples tested. For those instruments providing quantitative $\mathrm{HI}$ results, the mean intra-assay coefficient of variation (CVs) calculated for the triplicate determinations were $1.2 \%$ for the Modular System $\mathrm{P}$, $2.7 \%$ for the Integra 400 Plus, $0.1 \%$ for the ADVIA 2400 and $1.0 \%$ for the ADVIA 1800 , respectively.

\section{Discussion}

The receipt of hemolyzed samples in clinical laboratories is common and their identification is often difficult, especially when using arbitrary procedures such as the visual inspection. Detection of unsuitable specimens is even more challenging, if not impossible, when using whole blood specimens on point of care (POC) devices. Discrepant potassium results between POC devices and conventional laboratory instrumentation, rarely recorded, might be due to unrecognized in vitro hemolysis (11). Although quantitation of free hemoglobin in serum or plasma is theoretically possible by immunonephelometry (12), the use of this approach on all samples referred for testing is unpractical due to resultant increases in turnaround time, unavailability of the assay on instruments and uneconomical. Therefore, to simplify and standardize the process of identification of hemolyzed samples, and to evaluate their suitability for testing, automated detection of spectrophotometric interferents in patient serum and plasma caused by hemolysis, as well as by icterus and lipemia, have been developed. These systems consist of rapid, simple and inexpensive spectrophotometric blanked bichromatic measurements, most frequently between $405 \mathrm{~nm}$ and $700 \mathrm{~nm}$ (13). These flexible features are designed to provide users with an optional, automated and unbiased means of identification, so that unsuitable specimens can be dealt with according to the best laboratory practice. There is little doubt that the widespread implementation of the $\mathrm{HI}$, as well as other serum indexes, would be advantageous and profitable for a variety of reasons. These include the rapid detection of in vivo or in vitro hemolysis and the assessment of potential interference from hemo- 

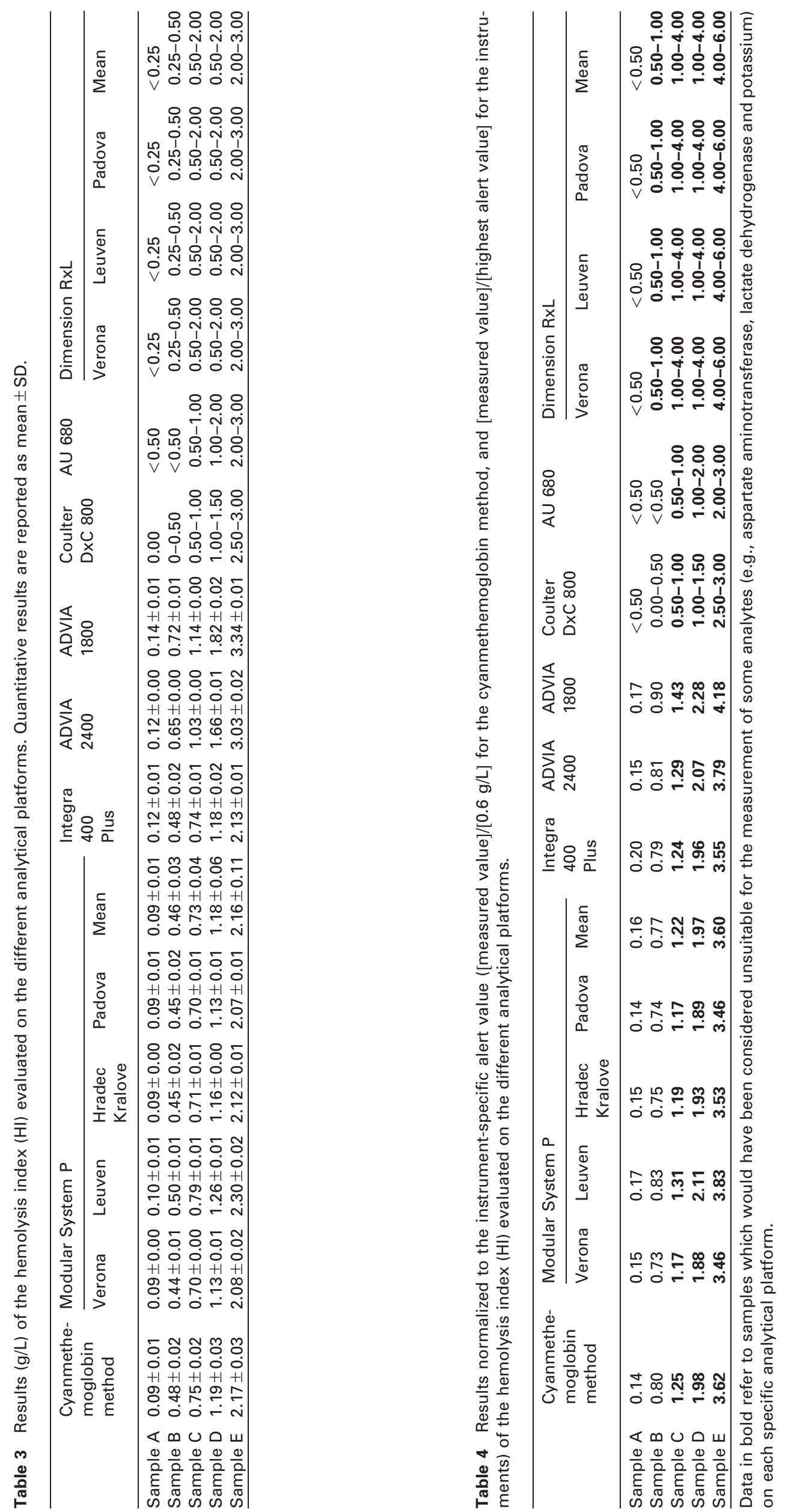
Table 5 Passing and Bablok regression analysis and Pearson correlation coefficient for the $\mathrm{HI}$ assayed on the four Roche Modular System P platforms.

\begin{tabular}{llll}
\hline & Padova & Leuven & Hradec Kralove \\
\hline Verona & $y=0.994 x$ & $y=1.104 x+0.01$ & $y=1.021 x$ \\
& $r=1.00 ; p<0.001$ & $r=1.00 ; p<0.001$ & $r=1.00 ; p<0.001$ \\
Padova & - & $y=1.111 x$ & $y=1.029 x-0.01$ \\
& - & $r=1.00 ; p<0.001$ & $r=1.00 ; p<0.001$ \\
Leuven & & - & $y=0.923 x$ \\
& & & $r=1.00 ; p<0.001$ \\
\hline
\end{tabular}

globin-based oxygen carriers (14), which would ultimately help diminish uncertainty in the preanalytical phase, enhance the quality in laboratory diagnostics, and reduce the chance of errors that can jeopardize the patient safety (7). Although the approach has been previously questioned (7), it is necessary to mention that the availability of quantitative results of hemoglobin in the samples might allow the use of formulas for correcting biases due to interference from hemolysis (15).

Although most preanalytical workstations and clinical chemistry platforms are now equipped with automatic systems for detecting, and some for quantitating interferents, no studies have previously assessed imprecision and reproducibility of these measures among facilities and, especially, among different instruments and manufacturers. The results of this multicenter evaluation demonstrate that overall imprecision of the instruments tested is satisfactory, as shown by inter-assay CVs between $0.1 \%$ and $2.7 \%$. We have also shown that the reproducibility among different facilities using the same instrument (Modular System P) is excellent. The overall instrumentspecific efficiency for identifying unsuitable samples was highly comparable, and no false negative results occurred when analyzing samples with free hemoglobin concentrations $>0.6 \mathrm{~g} / \mathrm{L}$. Likewise, all instruments except for the RxL Dimension correctly classified sample B, with a hemoglobin concentration of $0.048 \mathrm{~g} / \mathrm{L}$ as measured by the cyanmethemoglobin method, suitable for testing. These results are of particular significance in situations such as total laboratory automation or in laboratories where the preanalytical workstations are connected directly to the analytical platform and workflow makes visual inspection virtually impossible. However, we have also demonstrated that more efforts should be placed on standardization of reporting of HI. In addition to previous concerns that have been raised regarding the sometimes arbitrary and lack of evidence-based cutoffs to define the limits at which clinically significant interference starts (16), all the instruments that we tested provide either quantitative or qualitative results that are roughly comparable, but which should always be linked to the instrument-specific alert value for each test in order to harmonize and streamline their effectiveness (Tables 3 and 4). It is also important to highlight that it is necessary to evaluate the influence of hemolysis for each test and to state the limits of acceptability for each, as proposed in some validation protocols $(17,18)$. Unfortunately, we could not evaluate the $\mathrm{HI}$ on other instruments than those available to the participants of the study group. Thus, these results might not be universally applicable to other testing systems.

\section{Acknowledgements}

The authors wish to thank all the laboratory staff and technicians of the following clinical laboratories: Dr. Matteo Gelati (University Hospital, Verona, Italy), Dr. Martina Zaninotto (University Hospital, Padova, Italy), Dr. Elisabeth Lasnier and Dr. Nathalie Mario (Laboratoire de Biochimie Hôpital Saint Antoine, Paris, France), Dr. Lenka Zaloudkova and Dr. Magdalena Holeckova (University Hospital, Hradec Kralove, Czech Republic). The authors also wish to thank Miss. Sara Fernandez and Mr. Stephen Church for coordinating the study and organizing the shipment of the specimens.

\section{References}

1. Sonntag O. Haemolysis as an interference factor in clinical chemistry. J Clin Chem Clin Biochem 1986;24;12739.

2. Guder W. Haemolysis as an influence and interference factor in clinical chemistry. J Clin Chem Clin Biochem 1986;24:125-6.

3. Jones BA, Calam RR, Howanitz PJ. Chemistry specimen acceptability. A College of American Pathologists QProbes study of 453 laboratories. Arch Pathol Lab Med 1997;121:19-26.

4. Carraro P. Servidio P, Plebani M. Haemolyzed specimens: a reason for rejection or clinical challenge? Clin Chem 2000;46:306-7.

5. Bonini P, Plebani M, Ceriotti F, Rubboli F. Errors in laboratory medicine. Clin Chem 2002;48:691-8.

6. Lippi G, Guidi GC. Risk management in the preanalytical phase of laboratory testing. Clin Chem Lab Med 2007;

7. Lippi G, Blanckaert N, Bonini P, Green S, Kitchen S, Palicka $V$, et al. Haemolysis: an overview of the leading cause of unsuitable specimens in clinical laboratories. Clin Chem Lab Med 2008;46:764-72.

8. Lippi G, Salvagno GL, Montagnana M, Brocco G, Guidi GC. Influence of hemolysis on routine clinical chemistry testing. Clin Chem Lab Med 2006;44:311-6.

9. Lippi G, Banfi G, Buttarello M, Ceriotti F, Daves M, Dolci $A$, et al. Recommendations for detection and management of unsuitable samples in clinical laboratories. Clin Chem Lab Med 2007;45:728-36.

10. National Commmittee for Clinical Laboratory Standards. Reference and selected procedures for the quantitative 
determination of hemoglobin in blood: approved standard. 3rd Edition 2000. NCCLS document H15-A3. NCCLS, West Valley Rodad, Wayne, Pennsylvania, USA.

11. José RJ, Preller J. Near-patient testing of potassium levels using arterial blood gas analysers: can we trust these results? Emerg Med J 2008;25:510-3.

12. Lammers M. Gressner AM. Immunonephelometric quantification of free haemoglobin. J Clin Chem Clin Biochem 1987;25:363-7.

13. Glick MR, Ryder KW, Vroon DH, Masters BE, Sonntag O. Practical uses of serum indexes to reduce errors from lipemia, icterus, and hemolysis. Clin Chem 1990;36:1008.

14. Cameron SJ, Gerhardt G, Engelstad M, Young MA, Norris EJ, Sokoll LJ. Interference in clinical chemistry assays by the hemoglobin-based oxygen carrier, Hemospan. Clin Biochem 2009;42:221-4.
15. Vermeer HJ, Steen G, Naus AJ, Goevaerts B, Agricola PT, Schoenmakers $\mathrm{CH}$. Correction of patient results for Beckman Coulter LX-20 assays affected by interference due to hemoglobin, bilirubin or lipids: a practical approach. Clin Chem Lab Med 2007;45:114-9.

16. Steen G, Vermeer HJ, Naus AJ, Goevaerts B, Agricola PT, Schoenmakers $\mathrm{CH}$. Multicenter evaluation of the interference of hemoglobin, bilirubin and lipids on Synchron LX-20 assays. Clin Chem Lab Med 2006;44:413-9.

17. Vassault A, Grafmeyer D, Naudin C, Dumont G, Bailly M, Henny J, et al. Protocol for the validation of methods. Ann Biol Clin 1986;44:686-745.

18. Vassault A, Grafmeyer D, de Graeve J, Cohen R, Beaudonnet $A$, Bienvenu J. Quality specifications and allowable standards for validation of methods used in clinical biochemistry. Ann Biol Clin 1999;57:685-95. 